\title{
Optimization of Composite Plates with Spatially Varying Fiber Paths for Thermal Buckling
}

\author{
Adam V. Duran, ${ }^{*}$ Nicholas A. Fasanella ${ }^{\dagger}$ \\ University of Michigan, Ann Arbor, Michigan, 48109, USA \\ Veera Sundararaghavan ${ }^{\ddagger}$ and Anthony Waas ${ }^{\S}$ \\ University of Michigan, Ann Arbor, Michigan, 48109, USA
}

\begin{abstract}
Optimization of square composite laminates, with variable stiffness properties, for thermal buckling is presented. Spatially varying fiber paths produce material properties that are functions of position. In this work, a predefined fiber configuration is controlled by varying two global parameters; the angle at the edge and center of the plate. The critical buckling temperatures for such laminates are obtained numerically based on classical lamination theory and finite element method. The discretized domain simplifies analysis by transforming nonlinear fiber path functions to linear piecewise functions. Using this method, thermal responses for symmetric balanced laminates under constant thermal load is investigated. Optimal fiber angle configurations that maximize the critical buckling temperatures are obtained. Spatially varying fiber path configurations are found that increase the resistance of thermal buckling in comparison to straight fiber configurations.
\end{abstract}

\section{Nomenclature}

$N \quad$ Stress Resultant vector

$M \quad$ Moment Resultant vector

$\epsilon \quad$ Mid-plane Strain vector

$\kappa \quad$ Curvature vector

A Extensional Stiffness Matrix

$B \quad$ Coupling Stiffness Matrix

$D \quad$ Bending Stiffness Matrix

$U \quad$ Material Invariants

$h \quad$ Total Thickness of Plate

$t \quad$ Thickness single layer

$L \quad$ Total Length of Plate

$\theta \quad$ Fiber Angle

$\beta_{0} \quad$ Fiber Angle at Plate Center

$\beta_{1} \quad$ Fiber Angle at Plate Edge

$w \quad$ Out of Plane Displacement

$\bar{\theta} \quad$ Local Fiber Angle

$\Delta T$ Change in Temperature

$K_{M}$ Material Stiffness Matrix

$K_{G} \quad$ Geometric Stiffness Matrix

$\Delta T$ Change in Temperature

$\alpha \quad$ Coefficient of Thermal Expansion

E Young's Modulus

*PhD Candidate, Department of Aerospace Engineering, avduran@umich.edu, Student Member.

$\dagger$ PhD Candidate, Department of Aerospace Engineering, nickfas@umich.edu, Student Member.

¥Associate Professor, Department of Aerospace Engineering, veeras@umich.edu, and AIAA Member Grade.

$\S$ Felix Pawlowski Collegiate Professor, Department of Aerospace Engineering, dcw@umich.edu, and AIAA Member Grade. 


\section{Introduction}

Spacecrafts are subject to severe temperature fluctuations during flights. To maintain the structural integrity under high temperature environments, vehicle structural design concepts must differ from those of low Mach number aircrafts. In particular, management of high thermal stresses that occur during high speed flights involve development of materials that can withstand thermal bucking. Response of composite plate panels in compression (due to thermal loading and edge restraints) is a classical plate buckling problem. Earlier works have shown that composite plates offer superior buckling performance when compared to isotropic plates. Optimization methods and techniques have been applied to these material systems. Walker et. al. ${ }^{1}$ use a golden section method to find the optimal design for symmetric laminates subjected to nonuniform temperature distributions. In their work, plate geometry and boundary conditions are varied. Similarly, Topal et. al. ${ }^{2}$ investigate the effects of plate aspect ratios and boundary conditions on the optimal design configuration. A Modified Feasible Design optimization method is applied and antisymetric layups are considered. In general, for square symmetric balanced laminates, with simply sported boundary conditions, the the optimal configuration is given by $[ \pm 45]_{n s}$.

However, current technology allows us to relax the assumption that the fibers be straight for a laminated fiber composite. Advanced manufacturing techniques, such as fiber steering, allow fibers to be oriented along a desired path. Variable fiber path laminates produce unique boundary conditions that produce local transverse and compressive stresses which develop simultaneously. ${ }^{3}$ These stress fields may be exploited to increase buckling resistance in local areas where panels tend to buckle. Towards this end, Gürdal and Olmedo ${ }^{4-6}$ have developed material models for variable stiffness composites and have continued to, along with others, produce works for in-plane and buckling responses. Similar to straight fiber composites, optimization has been applied to these complex material systems. At the global level where the fiber path function is predefined, Negendra et. al. ${ }^{7}$ use a basis shape optimization approach to maximize critical buckling load for a plate with a hole. Similarly, Jegley et. al. ${ }^{8}$ optimize a curvilinear fiber path for a plate with a hole. In their work, they minimize stress concentrations using genetic discrete-valued optimizer. Alternatively, one could also optimize the fiber angle using a local parameterization of the fiber path. Hyer and Charette ${ }^{9}$ allow the fiber angle to vary at each element. In their work, they calculate the stress of each element and align fibers with the principal stress direction. Both Setoodeh et. al. ${ }^{10}$ and IJsselmuiden et. al. ${ }^{11}$ optimize the fiber angle locally at each node with respect to buckling and stiffness. As a result IJsselmuiden et. al. investigates the trade off between buckling and stiffness. Alternatively by minimizing the total thermal expansion, Rangarajan et. al. ${ }^{12}$ have shown that straight fibers paths are the optimum configuration to minimize total thermal expansion along principal material directions. In their work, a constant strain state is assumed and results are obtained analytically.

Although structural buckling of variable stiffness panels have been investigated, numerical optimization approaches applied specifically for thermal buckling performance is yet to be considered. In this work, this optimization problem is studied using a global parameterization of the fiber path, with the fiber path described by two design variables $\beta_{0}$ and $\beta_{1}$. The stacking sequence is restricted to symmetric and balanced $[ \pm \theta]_{n s}$. As an example, simply supported panels subjected to uniform temperature loading are investigates. The constitutive models are developed first, followed by buckling analysis of the out of plane equilibrium equation. The results are obtained numerically using an in-house finite element code for Eigen buckling analysis and validated with known results for straight fiber orientations. Finally, fiber paths are varied and the optimum configurations to resist thermal buckling are found using a gradient based optimization method. 


\section{Modeling}

\section{Material Model}

The constitutive relations for a thin laminate are based on classical lamination theory and are given in the form,

$$
\left\{\begin{array}{l}
N \\
M
\end{array}\right\}=\left[\begin{array}{ll}
A & B \\
B & D
\end{array}\right]\left\{\begin{array}{l}
\varepsilon \\
\kappa
\end{array}\right\}
$$

Here, the stiffness matrices are functions of panel position and are not constant. They are written compactly in the following form in terms of invariants ${ }^{4}$

$$
[A, B, D]=\left[\begin{array}{lll}
e_{11} & e_{12} & e_{16} \\
e_{12} & e_{22} & e_{26} \\
e_{16} & e_{26} & e_{66}
\end{array}\right]
$$

where

$$
\begin{gathered}
e_{11}=U_{1} V_{0}+U_{2} V_{1}+U_{3} V_{3} \\
e_{12}=U_{4} V_{0}-U_{3} V_{3}, \\
e_{16}=-\frac{1}{2} U_{2} V_{2}-U_{3} V_{4}, \\
e_{22}=U_{1} V_{0}-U_{2} V_{1}+U_{3} V_{3}, \\
e_{26}=-\frac{1}{2} U_{2} V_{2}+U_{3} V_{4}, \\
e_{66}=U_{5} V_{0}-U_{3} V_{3} .
\end{gathered}
$$

The invariants for an orthotropic material, $U_{i}$, are shown in Appendix A. The form of $V_{i}$ will change depending on the constitutive matrix. They are defined as,

$$
\begin{gathered}
V_{0(A, B, D)}=\left[h, 0, \frac{h^{3}}{12}\right] \\
V_{1(A, B, D)}=\int_{\frac{-h}{2}}^{\frac{h}{2}} \cos (2 \theta)\left[1, z, z^{2}\right] d z \\
V_{2(A, B, D)}=\int_{\frac{-h}{2}}^{\frac{h}{2}} \sin (2 \theta)\left[1, z, z^{2}\right] d z \\
V_{3(A, B, D)}=\int_{\frac{-h}{2}}^{\frac{h}{2}} \cos (4 \theta)\left[1, z, z^{2}\right] d z \\
V_{4(A, B, D)}=\int_{\frac{-h}{2}}^{\frac{h}{2}} \sin (4 \theta)\left[1, z, z^{2}\right] d z
\end{gathered}
$$

Here, $\theta$ is a function of position. For symmetric balanced laminates, the coupling stiffness matrix and the in-plane shear and normal coupling terms are eliminated; i.e. $B_{i j}=A_{16}=A_{26}=0$. Now there is no in-plane/bending coupling and Eq. 1 is reduced to,

$$
\left\{\begin{array}{c}
M_{x} \\
M_{y} \\
M_{x y}
\end{array}\right\}=\left[\begin{array}{ccc}
D_{11} & D_{12} & D_{16} \\
D_{12} & D_{22} & D_{26} \\
D_{16} & D_{26} & D_{66}
\end{array}\right]\left\{\begin{array}{c}
\kappa_{x} \\
\kappa_{y} \\
\kappa_{x y}
\end{array}\right\}
$$

Using Eq 2 - 5 the bending stiffness terms are defined by

$$
D_{11}=\frac{h^{3}}{12}\left(U_{1}+U_{2} \cos 2 \theta+U_{3} \cos 4 \theta\right)
$$




$$
\begin{gathered}
D_{12}=\frac{h^{3}}{12}\left(U_{4}-U_{3} \cos 4 \theta\right) \\
D_{16}=\frac{h^{3}}{12}\left(-\frac{1}{2} U_{2} \sin 2 \theta-U_{3} \sin 4 \theta\right) \\
D_{22}=\frac{h^{3}}{12}\left(U_{1}-U_{2} \cos 2 \theta+U_{3} \cos 4 \theta\right) \\
D_{26}=\frac{h^{3}}{12}\left(-\frac{1}{2} U_{2} \sin 2 \theta+U_{3} \sin 4 \theta\right) \\
D_{66}=\frac{h^{3}}{12}\left(U_{5}-U_{3} \cos 4 \theta\right)
\end{gathered}
$$

Note that the bending-twisting coupling terms $\left(D_{16}, D_{26}\right)$ are present.

\section{Fiber Path}

For simplicity, fibers are made to vary along one of the coordinate directions. This analysis may easily be extended for fibers that vary along multiple coordinate axes. A linear variation along the $x$-coordinate axis is achieved by defining the fiber orientation as,

$$
\theta(x)= \begin{cases}\frac{2\left(\beta_{1}-\beta_{0}\right)}{L} x+\beta_{0} & \text { for } 0 \leq x<\frac{L}{2} \\ \frac{2\left(\beta_{0}-\beta_{1}\right)}{L} x+\beta_{0} & \text { for }-\frac{L}{2} \leq x<0\end{cases}
$$

The angle $\beta_{1}$ is fiber angle at the edges of the plate $\left(x= \pm \frac{L}{2}\right)$, and $\beta_{0}$ is the angle of the fiber at the center of the plate $(x=0)$. The path of the fiber that passes through the origin as a function of $x$ is shown in Fig.1. Here the origin is located at the geometric center of the plate with a fiber angle $\beta_{0}=60^{\circ}$, while the angle at edge is $\beta_{1}=20^{\circ}$.

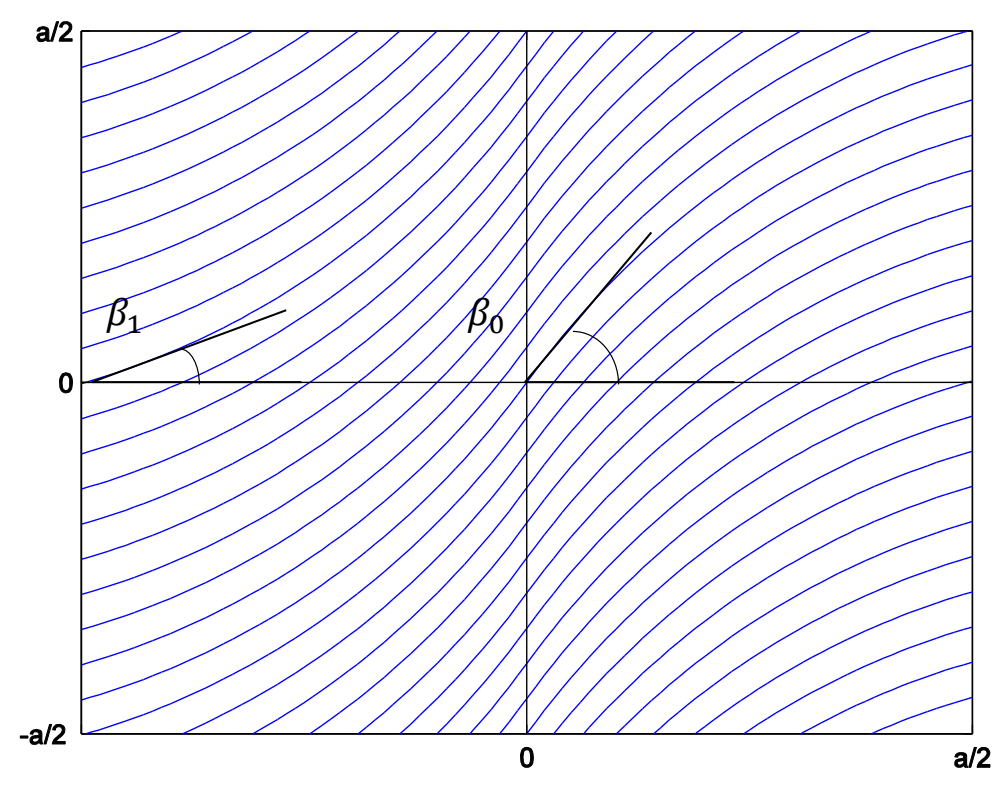

Figure 1. Curvilinear fiber path for $\beta_{1}=20^{\circ}$ and $\beta_{0}=60^{\circ}$. 


\section{Buckling Analysis}

For a simply supported symmetric balanced laminate, the Von Karman linearized out of plane buckling equation is given by,

$$
\begin{aligned}
-N_{x}^{i} \frac{\partial^{2} w}{\partial x^{2}}-N_{y}^{i} \frac{\partial^{2} w}{\partial y^{2}}-2 N_{x y}^{i} \frac{\partial^{2} w}{\partial x \partial y} & +D_{11} \frac{\partial^{4} w}{\partial x^{4}} \\
& +2\left(D_{12}+2 D_{16}\right) \frac{\partial^{4} w}{\partial x^{2} \partial y^{2}}+D_{22} \frac{\partial^{4} w}{\partial y^{4}} \\
& +4 D_{16} \frac{\partial^{4} w}{\partial x^{3} \partial y}+4 D_{26} \frac{\partial^{4} w}{\partial y^{3} \partial x}=0
\end{aligned}
$$

Entries denoted with superscript " $i$ " correspond to the pre-buckled configuration. The presence of the bending-twisting coupling terms $\left(D_{16}, D_{26}\right)$ in the Eq.8 makes it difficult to assume a series solution in solving this equation; therefore, the finite element method is used. For the finite element formulation, the discretized domain will produce locally straight, constant angle, fiber paths; each element will have a local fiber angle associated with it. Therefore, we model the global fiber orientation angle as a linear piecewise function. For this treatment, the local angle $\bar{\theta}$ is calculated by taking the elemental average of the angle $\theta$ (Eq 7). The local element laminate layup is then assumed to be $[ \pm \bar{\theta}]_{n s}$. Now the stiffness matrices $(A, B, D)$ are constant for each element. This process is shown in Fig.2 below. For an symmetric balanced laminate

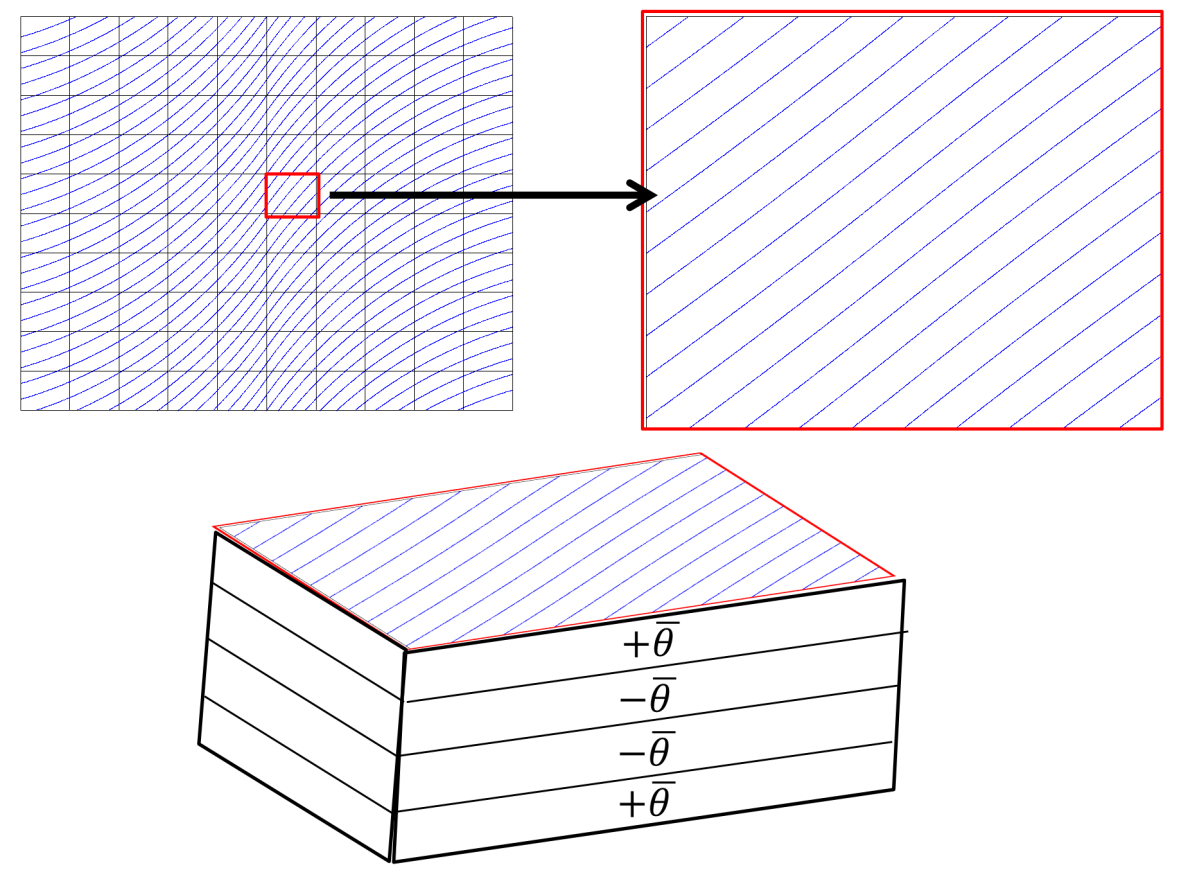

Figure 2. Curvilinear fiber path is approximated constant from each element.

with prescribed constant thermal load $\Delta T$, the pre-buckled loads are easily found using classical lamination theory.

$$
\begin{array}{r}
N_{x}^{i}=-\Delta T\left(A_{11} \alpha_{11}+A_{12} \alpha_{22}\right) \\
N_{y}^{i}=-\Delta T\left(A_{12} \alpha_{11}+A_{22} \alpha_{22}\right) \\
N_{x y}^{i}=0
\end{array}
$$

The critical buckling temperature $\Delta T_{\text {crit }}$ is determined using the following eigenvalue problem,

$$
\left|K_{M}+\lambda K_{G}\right|=0
$$


where the smallest eigenvalue $\lambda_{\min }$ corresponds to the critical buckling temperature $\Delta T_{\text {crit }}$. The material stiffness matrix $K_{M}$ and the geometric stiffness matrix $K_{G}$ are found using Galerkin method and are stated in Appendix B. This formulation uses 4-noded Kirchhoff plate elements ${ }^{13}$ with degrees of freedom $w, \partial w / \partial x$, and $\partial w / \partial y$.

\section{Validation}

\section{A. Cross-Ply Layup}

Validation is achieved by comparing results to straight angle configurations. For the first case, a four layer cross-ply layup $[0 / 90]_{s}$ is considered. The angles $\beta_{0}$ and $\beta_{1}$ are equal for straight fiber configurations. The critical buckling temperature found in reference ${ }^{14}$ is $\Delta T_{\text {crit }}=0.0996^{\circ} \mathrm{C}$. This work predicts a buckling temperature of $\Delta T_{\text {crit }}=0.0996^{\circ} \mathrm{C}$ and is in good agreement with the work cited.

\section{B. Angle-Ply Layup}

Next a Graphite/Epoxy angle-ply layup is considered. Material properties are given in Appendix $\mathrm{C}$ where $L=0.15 \mathrm{~m}$ and the $h=1.016 \mathrm{~mm}$. Again the angles $\beta_{0}$ and $\beta_{1}$ are equal and for a angle-ply layup $\beta_{0}=\beta_{1}=\theta$. Figure 3 below shows the critical buckling temperature distribution as a function of angle-ply angle $\theta$. For a four layer angle-ply layup this work predicts an optimal configuration of $[ \pm 45]_{s}$ which can be found as the optimal configuration to resist thermal buckling in other works. ${ }^{1,15}$

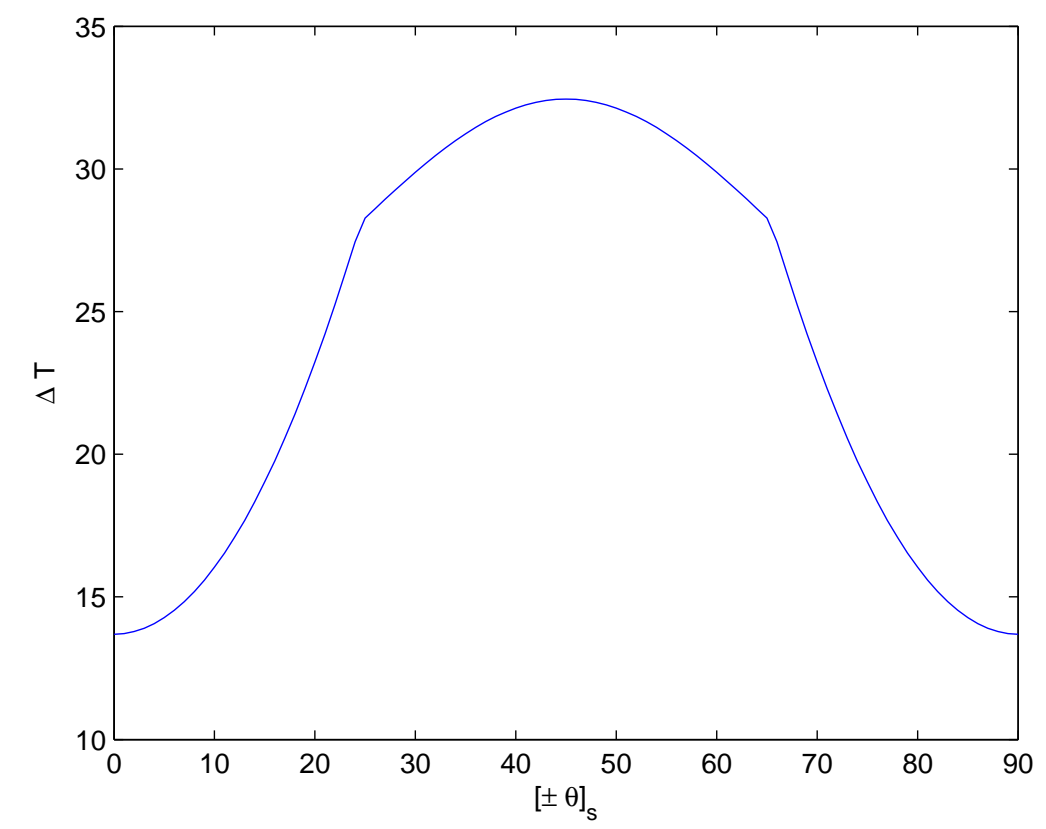

Figure 3. Critical buckling temperature distribution as a function of angle-ply angle.

\section{Complex Layup}

For the final case, a complex layup of $[+45 /-45 / 0 / 90]_{s}$ is considered. The same geometry and materials are used from the previous example. Results obtained numerically by a Rayleigh-Ritz formulation results in a critical buckling temperature of $\Delta T_{\text {crit }}=38.6^{\circ} \mathrm{C} .{ }^{15}$ This work predicts a higher critical buckling temperature of $\Delta T_{\text {crit }}=39.4^{\circ} \mathrm{C}$. Results from this section validate this method and analysis is continued with confidence. Now the fiber path is varied spatially for the same Graphite/Epoxy composite. 


\section{Results}

\section{A. Graphite/Epoxy Composite}

The critical buckling temperature for multiple configurations is found by performing an exhaustive search; the angles $\beta_{0}$ and $\beta_{1}$ are equally varied from $-90^{\circ}$ to $90^{\circ}$. A contour plot of the critical buckling temperature $\Delta T_{\text {crit }}$ as a function of the fiber angles $\beta_{0}$ and $\beta_{1}$ is shown in Fig. 4. These results show a temperature profile that contains multiple maxima and minima. Here, a diagonal across the figure represents a straight angle configuration $\left(\beta_{0}=\beta_{1}=\theta\right)$ where values in the positive domain reproduce results from Fig.3. The straight angle configuration with the largest resistance to thermal buckling is $[ \pm 45]_{s}$ corresponding to $\Delta T_{\text {crit }}=$ $32.45^{\circ} \mathrm{C}$. As expected the lowest critical buckling temperature corresponds to a $\beta_{1}=\beta_{0}=0$ orientation where all fibers are aligned in one direction, giving little buckling resistance in the direction perpendicular to the fibers. The temperature profile is skew symmetric and, therefore, is not unique over $-90^{\circ}$ to $90^{\circ}$ domain and $\Delta T\left(\beta_{0}, \beta_{1}\right)=\Delta T\left(-\beta_{0},-\beta_{1}\right)$. Although Fig.4 shows a complex temperature profile with multiple local maximums, it is obvious that a strong maximum exists.

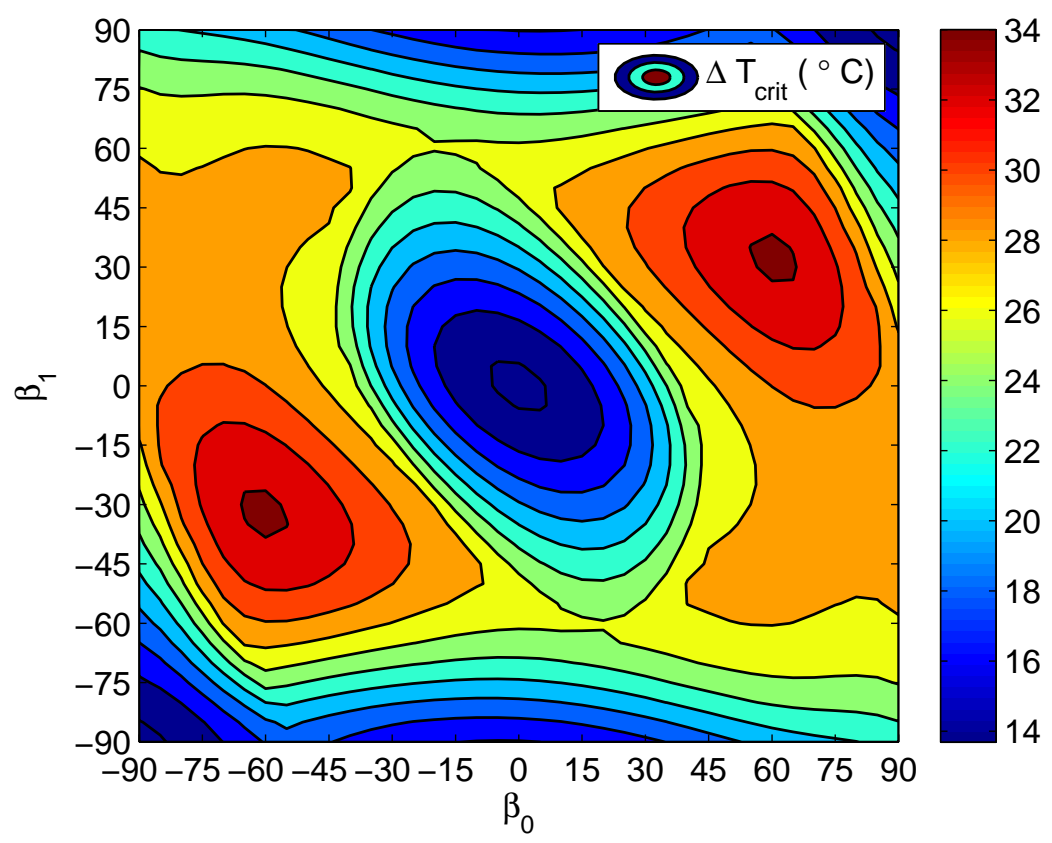

Figure 4. Temperature contour as a function of fiber angles for Graphite/Epoxy.

Finding the optimal fiber configuration that resists thermal buckling is a multi-variable optimization problem. A quasi-Newton method ${ }^{16}$ is implemented to find the maxima efficiently by maximizing $\Delta T_{\text {crit }}$ with respect to $\beta_{0}$ and $\beta_{1}$. Sensitivities are calculated using finite differences with a step size of $10^{-7}$. Fig. 5 shows the effect of the quasi-Newton method on the fiber path. The initial configuration of $[ \pm 45]_{s}$, where $\beta_{0}=\beta_{1}=\theta$, produces a straight fiber path. As the solution progresses, the fiber path begins to curve and becomes nonlinear. Fig.6 shows the progression of the solution on the contour of the temperature profile. The solution converges to the optimal configuration of $\beta_{0}=60.70^{\circ}$ and $\beta_{1}=32.19^{\circ}$ corresponding to the maximum critical buckling temperature of $\Delta T_{\text {crit }}=34.26^{\circ} \mathrm{C}$. Theses values give a $5.6 \%$ increase in thermal buckling load when compared to the optimum straight fiber configuration of $[ \pm 45]_{s}$.

\section{B. Angle Optimization for Multiple Materials}

Next, analysis is performed on multiple composite materials in an effort to further improve on percent increase of critical buckling temperature. Material constants are shown in Appendix C. Optimal results for these materials are reported on Table 1. The largest increase in critical buckling temperature is for Carbon/Epoxy where there is a 36.9\% increase in buckling temperature for $\beta_{1}=69.0^{\circ}$ and $\beta_{0}=-5.71^{\circ}$. Interestingly, since the fiber angle in not restricted, the optimal angle at the edge of the plate is negative 


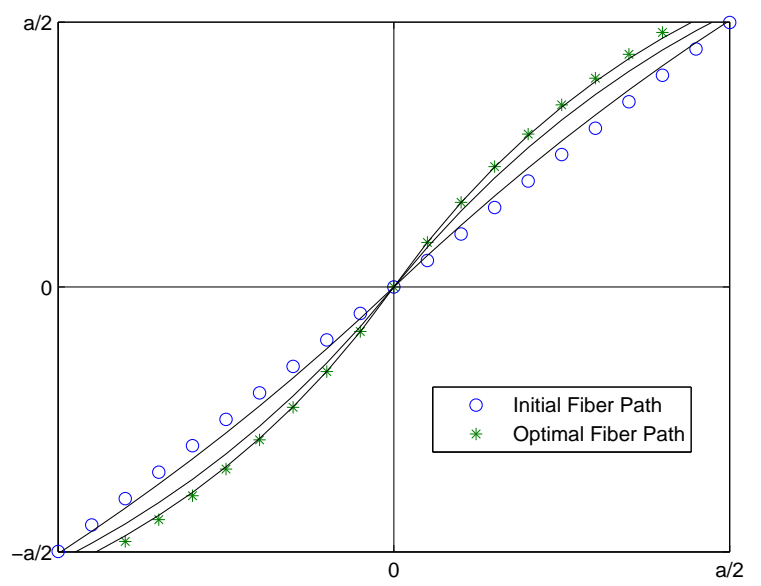

Figure 5. Quasi-Newton method effect on fiber path

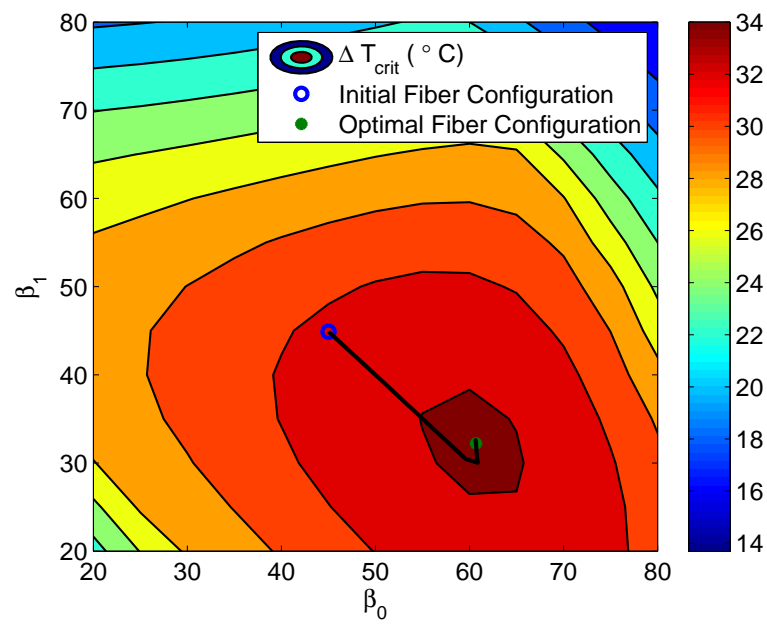

Figure 6. Quasi-Newton iterations to optimal angle configuration.

(Fig.7) or greater than $90^{\circ}$. This is also the case for the Boron/Epoxy (Fig.8) where the optimal angle at the center of the plate is negative. These result show that varying the fiber angle spatially provides a significant increase to resisting thermal buckling.

\begin{tabular}{ccccc}
\hline Material & $\beta_{0}$ & $\beta_{1}$ & $\Delta T_{\text {crit }}\left({ }^{\circ} \mathrm{C}\right)$ & {$[ \pm 45]_{s} \%$ increase } \\
\hline E-Glass/Epoxy & $6.710^{\circ}$ & $58.04^{\circ}$ & 5.58 & 2.8 \\
\hline S-Glass/Epoxy & $16.12^{\circ}$ & $54.74^{\circ}$ & 5.04 & 1.6 \\
\hline Kevlar/Epoxy & $66.05^{\circ}$ & $11.73^{\circ}$ & 22.18 & 24.1 \\
\hline Carbon/Epoxy & $69.00^{\circ}$ & $-5.705^{\circ}$ & 57.79 & 36.9 \\
\hline Carbon/Peek & $63.07^{\circ}$ & $29.50^{\circ}$ & 38.08 & 7.3 \\
\hline Carbon/Polyimide & $56.30^{\circ}$ & $36.68^{\circ}$ & 78.28 & 2.9 \\
\hline Boron/Epoxy & $-6.57^{\circ}$ & $63.28^{\circ}$ & 7.50 & 10.9 \\
\hline
\end{tabular}

Table 1. Optimization of fiber angle path for multiple materials

\section{Conclusion}

Thermal buckling of composite plates with spatially varying fiber orientations have been investigated. In this work square plates with simply supported boundary conditions, subjected to uniform temperature distribution, were considered. Optimization of the thermal buckling load was performed for multiple material models with respect to two fiber path parameters. It was found that curved fiber angle orientations provided better resistance to thermal buckling than straight fiber configurations in all cases. For the Carbon/Epoxy composite the optimal configuration obtained from this analysis provides a $36.9 \%$ increase to critical buckling temperature over the straight angle fiber configuration of $[ \pm 45]_{s}$. Future work includes a sensitivity analysis on the optimal fiber paths with respect to other material properties. This approach may be used in other work to investigate complex fiber paths with angles that vary in multiple spatial directions and for composite rectangular plates with varying layups. 


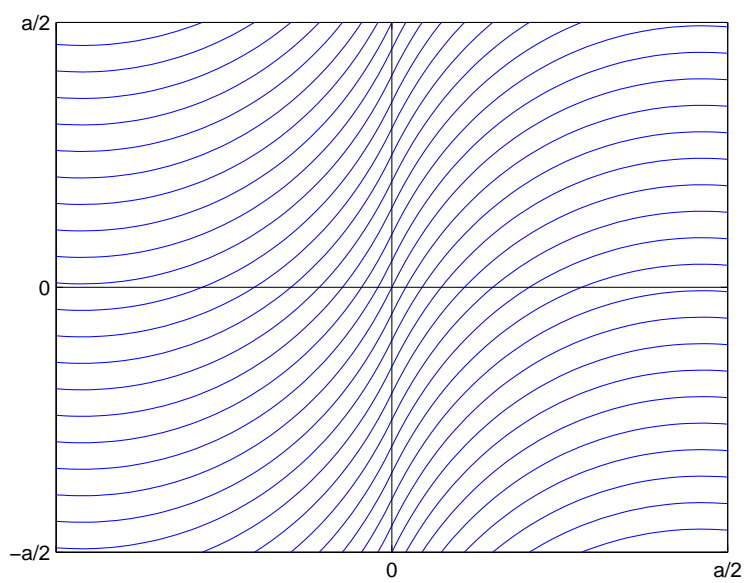

Figure 7. Optimum fiber path for Carbon/Epoxy.

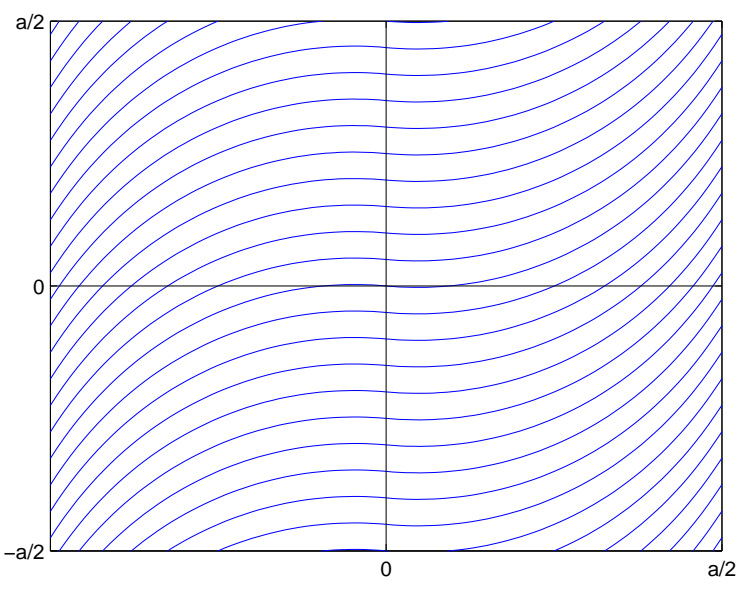

Figure 8. Optimum fiber path for Boron/Epoxy.

\section{Appendix}

\section{A. Orthotropic Invariants}

The invariants for an orthotropic material are given as

$$
\begin{array}{r}
U_{1}=\frac{3 Q_{11}+3 Q_{22}+2 Q_{12}+4 Q_{66}}{8} \\
U_{2}=\frac{Q_{11}-Q_{22}}{2} \\
U_{3}=\frac{Q_{11}+Q_{22}-2 Q_{12}-4 Q_{66}}{8} \\
U_{4}=\frac{Q_{11}+Q_{22}+6 Q_{12}-4 Q_{66}}{8} \\
U_{5}=\frac{Q_{11}+Q_{22}+2 Q_{12}-4 Q_{66}}{8}
\end{array}
$$

where

$$
\begin{aligned}
Q_{11}= & \frac{E_{11}}{1-\nu_{12} \nu_{21}} \\
Q_{12}= & \frac{\nu_{12} E_{22}}{1-\nu_{12} \nu_{21}} \\
Q_{22}= & \frac{E_{22}}{1-\nu_{12} \nu_{21}} \\
& Q_{66}=G_{12}
\end{aligned}
$$

\section{B. Stiffness Matrices}

The material stiffness matrix is given by

$$
K_{M}=\int_{y} \int_{x}[S]^{T}[D][S] t d x d y
$$


where $t$ is the element thickness, $D$ is the bending stiffness matrix (Eq.6) and

$$
S=\left\{\begin{array}{c}
\partial^{2} / \partial x^{2} \\
\partial^{2} / \partial y^{2} \\
2 \partial^{2} / \partial x \partial y
\end{array}\right\}[\phi]
$$

Here, the $\phi$ matrix is the shape functions corresponding to a 4-node Kirchhoff plate element. The geometric stiffness matrix is given by

$$
K_{G i j}=\int_{y} \int_{x}\left[\phi_{x}^{i} \frac{\partial \phi_{i}}{\partial x} \frac{\partial \phi_{j}}{\partial x}+\phi_{y}^{i} \frac{\partial \phi_{i}}{\partial y} \frac{\partial \phi_{j}}{\partial y}+\phi_{x y}^{i}\left(\frac{\partial \phi_{i}}{\partial x} \frac{\partial \phi_{j}}{\partial y}+\frac{\partial \phi_{i}}{\partial y} \frac{\partial \phi_{j}}{\partial x}\right)\right] t d x d y
$$

\section{Material Properties}

Material and thermal properties are given on Tables 2 and $3^{17}$

\begin{tabular}{ccccc}
\hline Material & $E_{1}(G P a)$ & $E_{2}(G P a)$ & $G_{12}(G P a)$ & $\nu_{12}$ \\
\hline Graphite/Epoxy & 155 & 8.07 & 4.55 & 0.22 \\
\hline E-Glass/Epoxy & 41 & 10.04 & 4.3 & 0.28 \\
\hline S-Glass/Epoxy & 45 & 11.0 & 4.5 & 0.29 \\
\hline Kevlar/Epoxy & 80 & 5.5 & 2.2 & 0.34 \\
\hline Carbon/Epoxy & 147 & 10.3 & 7.0 & 0.27 \\
\hline Carbon/Peek & 138 & 8.7 & 5.0 & 0.28 \\
\hline Carbon/Polyimide & 216 & 5.0 & 4.5 & 0.25 \\
\hline Boron/Epoxy & 201 & 21.7 & 5.4 & 0.17 \\
\hline
\end{tabular}

Table 2. Material properties

\begin{tabular}{ccc}
\hline Material & $\alpha_{1}\left({ }^{\circ} \mathrm{C}^{-1}\right) E-6$ & $\alpha_{2}\left({ }^{\circ} \mathrm{C}^{-1}\right) E-6$ \\
\hline Graphite/Epoxy & -0.07 & 30.1 \\
\hline E-Glass/Epoxy & 7.0 & 26 \\
\hline S-Glass/Epoxy & 7.1 & 30 \\
\hline Kevlar/Epoxy & -2.0 & 60 \\
\hline Carbon/Epoxy & -0.9 & 27 \\
\hline Carbon/Peek & -0.2 & 24 \\
\hline Carbon/Polyimide & 0.0 & 25 \\
\hline Boron/Epoxy & 6.1 & 30 \\
\hline
\end{tabular}

Table 3. Coefficients of thermal expansion

\section{Acknowledgments}

This material is based upon work supported by the National Science Foundation Graduate Research Fellowship Program Under DGE 1256260 


\section{References}

${ }^{1}$ Walker M., Reiss T., Adali S., and Verijenko V. E., "Optimal Design of Symmetrically Laminated Plates for Maximum Buckling Temperature," Journal of Thermal Stresses, Vol. 20, No. 1, 1997, pp. 21-33

${ }^{2}$ Topal U., and Uzman U., "Thermal Buckling Load Optimization of Laminated Composite Plates," Thin-Walled Structures, Vol. 46, No. 6, June 2008, pp. 667-675

${ }^{3}$ Gürdal Z., and Olmedo R. A., "Composite Laminates with Spatially Varying Fiber: Variable Stiffness Panel Concept," Proceedings of the AIAA/ASME/ASCE/AHS 33rd Structures, Structural Dynamics and Materials Conference, AIAA, Vol. 2, 1992, pp. 798-808

${ }^{4}$ Olmedo R. A., and Gürdal Z., "Buckling Response of Laminates with Spatially Varying Fiber Orientations," AIAA/ASME/ASCE/AHS/ASC 34th Structures, Structural Dynamics and Materials Conference, and AIAA/ASME Adaptive Structures Forum, La Jolla, CA, Apr. 19-22, 1993, Technical Papers. Pt. 4, pp. 2261-2269.

${ }^{5}$ Lopes C. S., Camanho P. P., and Gürdal Z., "Variable-Stiffness Composite Panels: Effects of Stiffness Variation on the Buckling and Failure Responses," 7th EuroMech Solid Mechanics Conference, Lisbon, Portugal, Sep. 7-11, 2009.

${ }^{6}$ Gürdal Z., Tatting B. F., and Wu C. K., "Variable-Stiffness Composite Panels: Effects of Stiffness Variation on the in-plane and Buckling Response," Composites Part A: Applied Science and Manufacturing, Vol. 39, No. 5, 2008, pp. 911-922

${ }^{7}$ Nagendra S., Kodiyalam S., and Davis J. E., "Optimization of Tow Fiber Paths for Composite Design," Proceedings of the 36th AIAA/ASME/ASCE/AHS/ASC Structures, Structural Dynamics and Materials Conference, New Orleans, LA., Apr. 1995.

${ }^{8}$ Jegley D. C., Tatting B. F., and Gürdal Z., "Optimization of Elastically Tailored Tow-placed Plates with Holes," Proceedings of the AIAA/ASME/ASCE/AHS/ASC 44th Structures, Structural Dynamics and Materials Conference, Norfolk, VA, Apr. 2003, pp. 2003-1420

${ }^{9}$ Hyer M. W., and Charette R. F., "Use of Curvilinear Fiber Format in Composite Structure Design," AIAA Journal, Vol. 29, No. 6, 1991, pp. 1011-1015.

${ }^{10}$ Setoodeh S., Abdalla M. M., IJsselmuiden S. T., and Gürdal Z., "Design of Variable-Stiffness Composite Panels for Maximum Buckling Load," Composite structures, Vol. 87, No. 1, 2009, pp. 109-117.

${ }^{11}$ IJsselmuiden S. T., Abdalla M. M., and Gürdal Z., "Optimization of Variable-Stiffness Panels for Maximum Buckling Load using Lamination Parameters," AIAA Journal, Vol. 48, No. 1, 2010, pp. 134-143.

${ }^{12}$ Rangarajan A., D'Mello R. J., Sundararaghavan V., and Waas A. M., "Minimization of Thermal Expansion of Symmetric, Balanced, Angle ply laminates by Optimization of Fiber Path Configurations," Composites Science and Technology, Vol. 71, No. 8, 31 May 2011, pp. 1105-1109

${ }^{13}$ Cook R. D., Malkus D. S., Plesha M. E, and Witt R. J., Concepts and Applications of Finite Element Analysis, $4^{\text {th }}$ ed., John Wiley and Sons, 2007, pp.573.

${ }^{14}$ Kant T., and Babu C. S., "Thermal Buckling Analysis of Skew Fibre-Reinforced Composite and Sandwich Plates using Shear Deformable Finite Element Models," Composite Structures, Vol. 49, No. 1, 2000, pp. 77-85.

${ }^{15}$ Meyers C.A., and Hyer C. A., "Thermally-induced, Geometrically Nonlinear Response of Symmetrically Laminated Composite Plates," Composites Engineering, Vol. 2, No. 1, Jan. 1992, pp. 3-20

${ }^{16}$ Fletcher R., and Powell M. J, "A Rapidly Convergent Descent Method for Minimization," The Computer Journal, Vol. 6, No. 2, 1963, pp. 163-168.

${ }^{17}$ Daniel I. M., and Ishai O., Engineering Mechanics of Composite Materials, Oxford University Press, New York, 2006, pp. $377-378$ 